\title{
Endoscopy-Assisted Laparoscopic Resection of the Gastric Wall Facilitated by a Double-Lifting Method
}

Small, early-stage gastric carcinomas and benign gastric tumors have been resected by endoscopic mucosal resection $(1,2)$ or by laparoscopy-assisted submucosal dissection (3). We describe here a new, endoscopy-assisted double-lifting laparoscopic resection of the gastric wall, which was used in two patients during removal of an early gastric carcinoma and a gastric lipoma.

The first patient was a 37-year-old man, who presented with weight loss. Panendoscopy demonstrated an early, depressed gastric carcinoma measuring $1.3 \mathrm{~cm}$ in diameter, located at the anterior wall of the gastric antrum (Figure 1). The second patient was a 65-year-old man suffering from epigastric pain. He was referred for endoscopy, which demonstrated an extrinsic, lobular mass on the anterior surface of the greater curvature of the gastric antrum. EUS showed a well-circumscribed intramural mass with a homogeneous echo pattern.

A laparoscopic approach was selected in both cases. After creation of a pneumoperitoneum, two 5-mm cannulas, two $10-\mathrm{mm}$ cannulas, and one $18-\mathrm{mm}$ cannula were inserted into the peritoneal cavity. Via one of the 5-mm ports, two special lifting needles were inserted into the abdomen. The endoscope visualized the lesions and the sites on the stomach wall to which the lifting needles had to be passed. The polypectomy grasping forceps pulled the needles through the gastric wall and positioned them around the lesions. After the gastric wall had been elevated, the lesions were resected through the $18-\mathrm{mm}$ port with an endoscopic linear cutter (Ethicon ELC-60). The histological examinations revealed a well-differentiated carcinoma located in the mucosal layer of the stomach in the first patient, and a lipoma in the second.

The advantages of this endoscopically positioned double-lifting method are that the affected gastric wall can be removed safely at a distance from the lesion, and all layers of the gastric wall are available for histological examination (Figure 2). This technique is strongly recommended, since adenocarcinoma may occur underneath a submucosal lipoma (4).

A. Altorjay' ', I. Szántó ', J. Garcia', P. Nagy², J. Kiss'

'Dept. of Surgery

${ }^{2}$ Institute of Pathology, Postgraduate Medical School,

Imre Haynal University of Health Sciences, Budapest, Hungary

\section{References}

1. Takechi K. A modified technique for endoscopic mucosal resection of small early gastric carcinomas. Endoscopy 1992; 24: $232-8$.

2. Kitano S, Shimoda K, Miyahara M, et al. Laparoscopic approaches in the management of patients with early gastric carcinomas. Surg Laparosc Endosc 1995; 5: 359-62.

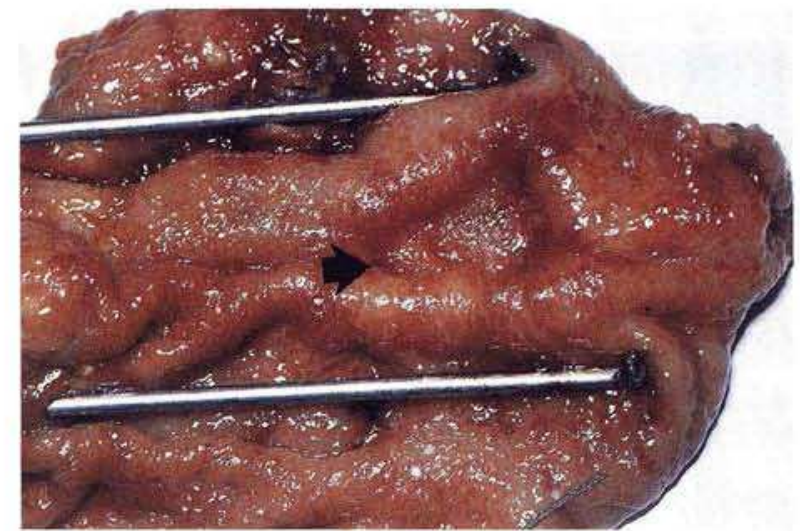

Figure 1: Resected specimen of an early, depressed gastric carcinoma, with the endoscopically positioned lifting needles.

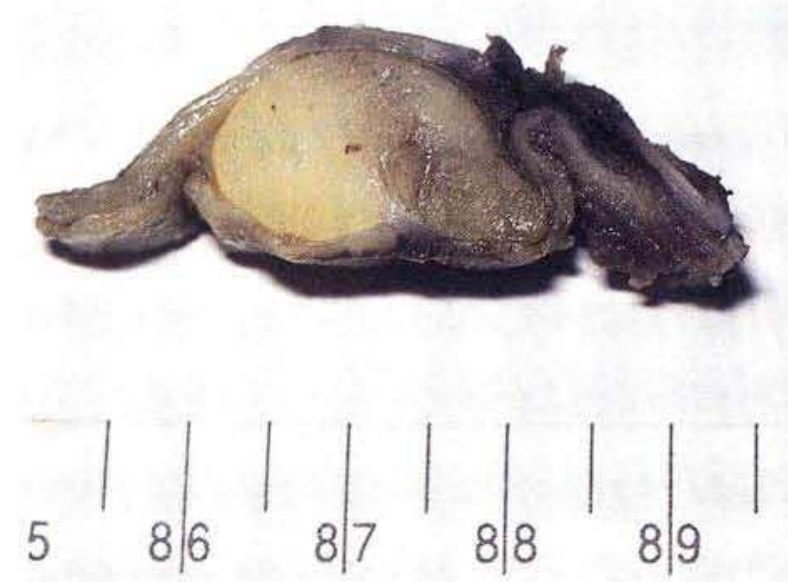

Figure 2: Resected specimen of the laparoscopically removed gastric lipoma.

3. Lacy AM, Tabet J, Grande L, et al. Laparoscopic-assisted resection of gastric lipoma. Surg Endosc 1995; 9: 995-7.

4. Moreira LF, Iwagaki H, Matsuno T, et al. Submucosal lipoma synchronous with an early gastric cancer. J Clin Gastroenterol 1992; 14: $173-82$.

Corresponding Author

Á. Altorjay, M.D., Ph.D., Krisztina krt. 69

1016 Budapest, Hungary 